\title{
Impact of sample preparation upon intracellular metabolite measurements in 3D cell culture systems
}

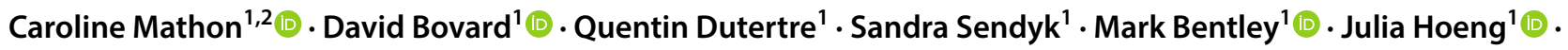 \\ Arno Knorr ${ }^{1}$
}

Received: 3 January 2019 / Accepted: 29 May 2019 / Published online: 12 June 2019

(c) The Author(s) 2019

\begin{abstract}
Introduction Interest in cell culture metabolomics has increased greatly in recent years because of its many potential applications and advantages (e.g., in toxicology). The first critical step for exploring the cellular metabolome is sample preparation. For metabolomics studies, an ideal sample preparation would extract a maximum number of metabolites and would enable reproducible, accurate analysis of a large number of samples and replicates. In addition, it would provide consistent results across several studies over a relatively long time frame.

Objectives This study was conducted to evaluate the impact of sample preparation strategies on monitoring intracellular metabolite responses, highlighting the potential critical step(s) in order to finally improve the quality of metabolomics studies. Methods The sample preparation strategies were evaluated by calculating the sample preparation effect, matrix factor, and process efficiency (PE) for 16 tobacco exposition-related metabolites, including nicotine, nicotine-derived nitrosamine ketone, their major metabolites, and glutathione, using isotopically-labelled internal standards. Samples were analyzed by liquid chromatography (LC) coupled to high-resolution mass spectrometry (HRMS).

Results A sample drying step increased losses or variability for some selected metabolites. By avoiding evaporation, good sample preparation recovery was obtained for these compounds. For some metabolites, the cell or culture type impacted PE and matrix factor.

Conclusion In our sample preparation protocol, the drying-reconstitution step was identified as the main cause of metabolite losses or increased data variability during metabolomics analysis by LC-HRMS. Furthermore, PE was affected by the type of matrix. Isotopologue internal standards fully compensate losses or enhancements.
\end{abstract}

Keywords Cell culture $\cdot$ Effectiveness $\cdot$ Extraction $\cdot$ Intracellular metabolites $\cdot$ LC-HRMS $\cdot$ Sample preparation

\section{Introduction}

Characterization of the cellular metabolome, represented by intermediates and end products of all enzymatic reactions in a cell, enables the evaluation of cellular biochemical activity/networks. This information can predict molecular physiology and phenotypic changes in an organism (Töpfer et al. 2015). Stimuli or changes (e.g., environment, virus, food intake, smoking) induce responses at the metabolite level

Caroline Mathon

Caroline.Mathon@chuv.ch

1 PMI R\&D, Philip Morris Products S.A., Quai Jeanrenaud 5, 2000 Neuchâtel, Switzerland

2 Present Address: Unit of Toxicology, CURML, LausanneGeneva, Switzerland because of upstream modifications to DNA, mRNA, and proteins (Ivanisevic et al. 2013; Vinaixa et al. 2016; Worley and Powers 2013).

Metabolomics approaches may be categorized as follows: (i) An untargeted approach encompasses as many metabolites as possible to provide a comprehensive analysis. For these global analyses, the entire workflow must be nonselective, reproducible, and unbiased in order to preserve the integrity of cellular metabolites and include as many metabolites as possible (Cala and Meesters 2017; Ulmer et al. 2015). Metabolite abundances or relative responses are reported. (ii) A targeted approach is focused on a limited list of metabolites. In this case, sample preparation and analytical methods are optimized for specific compounds, which are absolutely quantified using calibration curves and internal standards for each metabolite. (iii) A semi-targeted approach 
lies between the targeted and untargeted approaches. Many metabolites are screened and semi-quantified, using single calibration curves for multiple compounds (Broadhurst et al. 2018).

For toxicological assessment, changes in metabolic profiles and their relationship to various biochemical pathways are investigated. Untargeted approaches have been used for this purpose, for instance, to explore the global impact of environmental chemical contaminants on earthworms (Griffith et al. 2018) or on human cells (Hartung et al. 2017), or to assess the impact of tobacco exposure on in vitro systems (Iskandar et al. 2017; Kogel et al. 2016; Zanetti et al. 2017). For in vitro toxicology studies, two-dimensional (2D) cell cultures have been the most commonly investigated systems for decades. Currently, however, human three-dimensional (3D) tissue cultures are widely regarded as more relevant than 2D monolayer cell cultures for in vitro toxicological assessments. In 2D culture systems, the extracellular matrix and cell-cell and cell-matrix interactions, all essential for differentiation, proliferation, and cellular functions, are not present. In contrast, 3D cultures are sometimes composed of several cell types where cell-cell and cell-matrix interactions are formed, recreating physiological tissue structure, enabling cell differentiation, and finally, potentially increasing drug susceptibility (Bovard et al. 2017; Ravi et al. 2015). Several 3D tissues mimicking human organs are commercially available or can be generated by investigators in-house. Bronchial 3D organotypic tissues can be prepared starting from primary airway epithelial human cells grown at the air-liquid interface (ALI) and seeded on a collagen-based support: in these conditions, cells differentiate to form a functional bronchial tissue composed of basal, goblet, and ciliated cells (Fig. 1a) (Karp et al. 2002). Such 3D bronchial tissue models have morphological and metabolic characteristics that are comparable with those of large human airways (Baxter et al. 2015; Pezzulo et al. 2011). Furthermore, when bronchial tissue cultures are developed and maintained at the ALI, they can be exposed to various aerosols, including cigarette smoke or a candidate modified risk tobacco product aerosol, mimicking in vivo inhalation exposure (Iskandar et al. 2017). These toxicological investigations have greatly improved over the last few years thanks to the development of new 3D models that better mimic lung function. In addition to bronchial tissues, liver spheroids were also investigated, as the liver is the most metabolically active organ (Fathi et al. 2017). Liver function can be mimicked by liver spheroids composed of HepaRG ${ }^{\mathrm{TM}}$ cells (Fig. 1b). HepaRG ${ }^{\mathrm{TM}}$ cells are terminally differentiated hepatic cells derived from a human hepatic progenitor cell line, retaining many characteristics of primary human hepatocytes. The spheroid organization enables close contact between cells as well

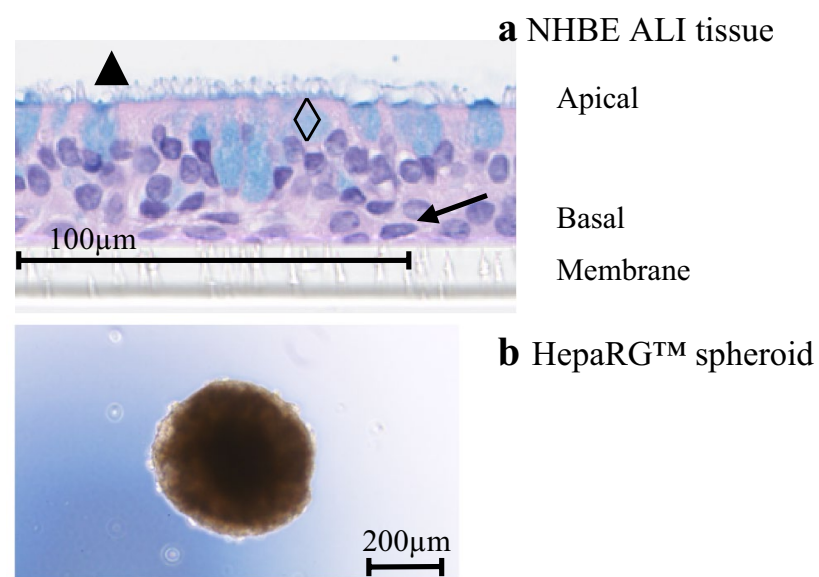

Fig. 1 a Representative histological section of NHBE ALI tissues, stained with hematoxylin, eosin, and Alcian blue. Magnification $\times 20$. The in vitro tissue was composed of ciliated cells (black triangle), goblet cells (empty diamond), and basal cells (black arrow). b Representative bright-field images of HepaRG ${ }^{\mathrm{TM}}$ spheroid. Magnification $\times 10$

as polarization and formation of bile canaliculi (Alepee et al. 2014). In addition to these morphological advantages, 3D organization of liver cells leads to increased albumin secretion, the ability for cytochrome P450 family induction and longer cell survival than 2D cultures (Takahashi et al. 2015). As described by Groell et al. 3D cell cultures represent a perfect compromise between $2 \mathrm{D}$ and animal models (Groell et al. 2018).

For untargeted cellular metabolome characterization, sample preparation must be non-discriminative and precise. The protocol must also maintain sample stability, because intracellular metabolites can be rapidly degraded or metabolized by enzymatic reactions, and must minimize contaminants in both sample and detector (Drouin et al. 2018; Pinu et al. 2017). Precise results from single-time-point analyses or kinetic studies are necessary in order to obtain an accurate snapshot of the metabolome. Cell extracts must be rapidly and reproducibly quenched to stop metabolic activity to ensure consistent results across many samples and several studies over an extended time frame. Compound loss or modification caused by chemical or enzymatic reactions occurring during sample harvest, preparation, and analysis must be minimized. Changes occurring in the autosampler during chromatographic analysis can be monitored by comparing the signal responses with injection order and can be corrected using quality control (QC) samples or by including appropriate stable isotopically labelled internal standards (ISTD) (Broadhurst et al. 2018). ISTDs can be used to compensate for changes occurring at any time after they are added to the samples, even during sample preparation (Pinu et al. 2017). It is of paramount importance to minimize potential changes before the addition of ISTDs, which are 
difficult to evaluate, by decreasing duration of sampling and by placing samples into low temperature storage as soon as possible (Pinu et al. 2017).

To our knowledge, there are no harmonized procedures for sample preparation of 3D cell cultures for untargeted metabolomics. More generally, standard protocols for sample preparation have not been fully established, mostly because metabolomics is a rapidly evolving technique utilizing a broad range of analytical techniques and sample matrices and measuring metabolites with a wide variety of physical and chemical properties (Bi et al. 2013; Daskalaki et al. 2018; Ulmer et al. 2015). Nevertheless, one recent guideline for untargeted metabolomics analysis is available (Broadhurst et al. 2018). Although sample preparation is a crucial step, as it can affect the entire study outcome, it is often neglected, and evaluation of its impact is rarely considered (Cuykx et al. 2017; Dettmer et al. 2011; Martinovic et al. 2018; Römisch-Margl et al. 2012; Sapcariu et al. 2014). Intracellular metabolites were extracted using freezethawing cycles or homogenization, with and without a drying-reconstitution step. Sample extracts were analyzed by liquid chromatography (LC) coupled to high-resolution mass spectrometry (HRMS). To evaluate the reproducibility of the generic analytical approach, we chose 16 chemicals and eight ISTDs specific to our research area. These compounds (Table 1), all related to tobacco exposure, included nicotine, nicotine-derived nitrosamine ketone (NNK), and their major metabolites as well as glutathione (GSH), a biomarker of oxidative stress. Of these, nicotine, NNK, and their metabolites are either abundant in tobacco-derived aerosols (nicotine) or have carcinogenic potential (NNK) (Konstantinou et al. 2018). Understanding the metabolism pathways for xenobiotics, such as nicotine and its metabolites, the major markers for cigarette smoke exposure, requires precise metabolite analyses, either at individual time points or in kinetic studies. Based on this compound pallet, method was evaluated by calculating the effect of the sample preparation, matrix factor, and process efficiency (PE) for the selected metabolites and ISTDs for bronchial and hepatic 3D cell cultures.

\section{Materials and methods}

\subsection{Reagents and chemicals}

LC coupled to mass spectrometry (MS)-grade water and methanol (LC/MS Chromasolv) were supplied by Honeywell Riedel-de Haën ${ }^{\mathrm{TM}}$ (Seelze, Germany). Analytical standards, ISTDs, and formic acid were supplied by Sigma Aldrich (St. Louis, MO, USA), Toronto Research Chemicals (Ontario, Canada), TLC PharmaChem (Ontario, Canada), or Santa Cruz Biotechnology (Dallas, TX, USA), depending on availability (details for analytes and ISTDs are presented in Table 1).

The standard and ISTD solutions (commercially obtained or prepared in the laboratory from powder), at approximately $1 \mathrm{mg} / \mathrm{mL}$, were stored at $-20{ }^{\circ} \mathrm{C}$ and diluted in methanol/water $(4: 1 \mathrm{v} / \mathrm{v})$ on the day of analysis for spiking mixtures and calibration curves. Calibration levels ranged over three orders of magnitude for each metabolite, and concentrations were selected based on MS sensitivity of each metabolite and its expected concentrations in the samples.

\subsection{HepaRG $^{\mathrm{TM}}$ spheroids}

HepaRG ${ }^{\mathrm{TM}}$ cells (Ref. HPRGC10, ThermoFisher Scientific) were first thawed in William's E medium (Ref. 12551032, ThermoFisher) supplemented with GlutaMAX $^{\mathrm{TM}}$ (Ref. 35050061, ThermoFisher) and HepaRG $^{\text {TM }}$ Thaw, Plate \& General Purpose Medium Supplement (Ref. HPRG770, ThermoFisher). After thawing, 25,000 cells were seeded in each well of an ultra-low adhesion 96-well plate (Ref. 4520, Corning, NY, USA). The 96-well plates were kept in the incubator at $37^{\circ} \mathrm{C}$ for 4 days before medium replacement. On the fourth day, cells formed dense aggregates with a visible extracellular matrix confirming spheroid formation. Thereafter, the cell culture medium was renewed every $2-3$ days. Spheroids were used once mature, approximately 1 week after thawing HepaRG ${ }^{\mathrm{TM}}$ cells.

\subsection{NHBE ALI tissues}

Normal human bronchial epithelial (NHBE) ALI tissues were prepared following a procedure described by STEMCELL Technologies (PneumaCult ${ }^{\mathrm{TM}}$ Medium, Document \#29252; STEMCELL Technologies, Vancouver, Canada). Briefly, NHBE cells (Lonza, Basel, Switzerland) were cultured in T75-flasks using PneumaCult ${ }^{\mathrm{TM}}$-EX PLUS medium (Ref. 05040, STEMCELL Technologies) at $37{ }^{\circ} \mathrm{C}$ with $5 \% \mathrm{CO}_{2}$ and $90 \%$ relative humidity. Once the cells were $80 \%$ confluent, they were detached from the flask using trypsin-ethylenediaminetetraacetic acid (EDTA) (Ref. CC-5034, Lonza), and 50,000 cells were seeded on a Collagen I-coated Transwell@ insert (Corning ${ }^{\circledR}$, Corning, NY, USA). Both apical and basal sides of the inserts were filled with PneumaCult ${ }^{\mathrm{TM}}-\mathrm{EX}$ PLUS medium and maintained for 3 days. Subsequently, the culture was airlifted by removing the apical medium; the basal medium was replaced with the PneumaCult ${ }^{\mathrm{TM}}$-ALI medium (STEMCELL Technologies). Tissues were used for experiments starting from day 28 after airlift. 
Table 1 List of the major nicotine, NNK, and GSH metabolites investigated, with their corresponding abbreviations, ISTDs, CAS number, formula, molecular ion $\mathrm{m} / \mathrm{z}$ in positive electrospray ionization, retention times, and suppliers

\begin{tabular}{|c|c|c|c|c|c|c|c|c|}
\hline Categories & Metabolites & Abbreviations & ISTDs & CAS & Formula & $m / z[\mathrm{M}+\mathrm{H}]^{+}$ & $t_{R}$ & Suppliers \\
\hline \multirow[t]{12}{*}{ Nicotine } & Cotinine $\mathrm{D}_{3}$ & Cot $D_{3}$ & N/A & $110952-70-0$ & $\mathrm{C}_{10} \mathrm{H}_{5} \mathrm{D}_{7} \mathrm{~N}_{2} \mathrm{O}$ & 180.12107 & 8.0 & Sigma-Aldrich \\
\hline & $\begin{array}{l}\text { 3-hydroxy-cotinine- } O-\beta \text { - } \\
\text { glucuronide }\end{array}$ & OH Cot- $O$-gluc. & $\operatorname{Cot} \mathrm{D}_{3}$ & $132929-88-5$ & $\mathrm{C}_{16} \mathrm{H}_{20} \mathrm{~N}_{2} \mathrm{O}_{8}$ & 369.12924 & 3.9 & $\mathrm{TRC}$ \\
\hline & Cotinine & Cot & $\operatorname{Cot} \mathrm{D}_{3}$ & $486-56-6$ & $\mathrm{C}_{10} \mathrm{H}_{12} \mathrm{~N}_{2} \mathrm{O}$ & 177.10224 & 8.0 & Sigma-Aldrich \\
\hline & Cotinine- $N$-oxide & Cot- $N-\mathrm{Ox}$ & $\operatorname{Cot} \mathrm{D}_{3}$ & $36508-80-2$ & $\mathrm{C}_{10} \mathrm{H}_{12} \mathrm{~N}_{2} \mathrm{O}_{2}$ & 193.09715 & 7.5 & TLC PharmaChem \\
\hline & Nicotine $\mathrm{D}_{3}$ & Nic $D_{3}$ & N/A & $69980-24-1$ & $\mathrm{C}_{10} \mathrm{D}_{3} \mathrm{H}_{11} \mathrm{~N}_{2}$ & 166.14181 & 8.4 & Sigma-Aldrich \\
\hline & Nicotine & $\mathrm{Nic}$ & $\mathrm{Nic}_{3}$ & $54-11-5$ & $\mathrm{C}_{10} \mathrm{H}_{14} \mathrm{~N}_{2}$ & 163.12298 & 8.4 & Sigma-Aldrich \\
\hline & $\begin{array}{l}\text { Nicotine- } N-\beta- \\
\text { glucuronide } \mathrm{D}_{3}\end{array}$ & Nic-N-gluc. $\mathrm{D}_{3}$ & N/A & $329002-74-6$ & $\mathrm{C}_{16} \mathrm{H}_{19} \mathrm{D}_{3} \mathrm{~N}_{2} \mathrm{O}_{6}$ & 342.17389 & 1.8 & SCBT \\
\hline & Nicotine- $N$-glucuronide & Nic- $N$-gluc. & Nic- $N$-Gluc. $\mathrm{D}_{3}$ & $152306-59-7$ & $\mathrm{C}_{16} \mathrm{H}_{22} \mathrm{~N}_{2} \mathrm{O}_{6}$ & 339.15506 & 1.8 & TRC \\
\hline & Nicotine- $N$-oxide $\mathrm{D}_{3}$ & Nic- $N$-ox $\mathrm{D}_{3}$ & N/A & $491-26-9$ & $\mathrm{C}_{10} \mathrm{H}_{11} \mathrm{D}_{3} \mathrm{~N}_{2} \mathrm{O}$ & 182.13672 & 8.8 & TLC PharmaChem \\
\hline & Nicotine- $N$-oxide & Nic- $N$-ox & Nic- $N$-ox $\mathrm{D}_{3}$ & $491-26-9$ & $\mathrm{C}_{10} \mathrm{H}_{14} \mathrm{~N}_{2} \mathrm{O}$ & 179.11789 & 8.8 & TLC PharmaChem \\
\hline & Nornicotine $\mathrm{D}_{4}$ & Nornic $\mathrm{D}_{4}$ & N/A & $66148-18-3$ & $\mathrm{C}_{9} \mathrm{H}_{8} \mathrm{D}_{4} \mathrm{~N}_{2}$ & 153.13243 & 6.0 & TRC \\
\hline & Nornicotine & Nornic & Nornic $\mathrm{D}_{4}$ & $5746-86-1$ & $\mathrm{C}_{9} \mathrm{H}_{12} \mathrm{~N}_{2}$ & 149.10732 & 6.0 & Sigma-Aldrich \\
\hline \multirow[t]{10}{*}{ NNK } & $\begin{array}{l}\text { 4-(Methylnitrosamino)- } \\
\text { 1-(3-pyridyl)-1- } \\
\text { butanol } \mathrm{D}_{3}\end{array}$ & $\mathrm{NNAL} \mathrm{D}_{3}$ & N/A & $1020719-61-2$ & $\mathrm{C}_{10} \mathrm{H}_{12} \mathrm{D}_{3} \mathrm{~N}_{3} \mathrm{O}_{2}$ & 213.14253 & 8.4 & TRC \\
\hline & $\begin{array}{l}\text { 4-(Methylnitrosamino)- } \\
\text { 1-(3-pyridyl)-1- } \\
\text { butanol }\end{array}$ & NNAL & $\mathrm{NNAL} \mathrm{D}_{3}$ & 76014-81-8 & $\mathrm{C}_{10} \mathrm{H}_{15} \mathrm{~N}_{3} \mathrm{O}_{2}$ & 210.12370 & 8.4 & TRC \\
\hline & $\begin{array}{l}\text { 4-(Methylnitrosamino)- } \\
\text { 1-(3-pyridyl)-1- } \\
\text { butanone }\end{array}$ & NNK & $\mathrm{NNAL} \mathrm{D}_{3}$ & 64091-91-4 & $\mathrm{C}_{10} \mathrm{H}_{13} \mathrm{~N}_{3} \mathrm{O}_{2}$ & 208.10805 & 11.7 & Sigma-Aldrich \\
\hline & $\begin{array}{l}\text { 4-(Methylnitrosamino)- } \\
\text { 1-(3-pyridyl- } N \text {-oxide)- } \\
\text { 1-butanol }\end{array}$ & NNAL- $N$-ox & $\mathrm{NNAL} \mathrm{D}_{3}$ & $85352-99-4$ & $\mathrm{C}_{10} \mathrm{H}_{15} \mathrm{~N}_{3} \mathrm{O}_{3}$ & 226.11862 & 8.2 & TRC \\
\hline & $\begin{array}{l}\text { 4-(Methylnitrosamino)- } \\
\text { 1-(3-pyridyl- } N \text {-oxide)- } \\
\text { 1-butanone }\end{array}$ & NNK- $N$-ox & $\mathrm{NNAL} \mathrm{D}_{3}$ & $76014-82-9$ & $\mathrm{C}_{10} \mathrm{H}_{13} \mathrm{~N}_{3} \mathrm{O}_{3}$ & 224.10297 & 9.3 & $\mathrm{TRC}$ \\
\hline & $\begin{array}{l}\text { 4-Hydroxy-1-(3- } \\
\text { pyridyl)-1-butanone } \\
\mathrm{D}_{2}\end{array}$ & $\mathrm{HPB} \mathrm{D}_{2}$ & N/A & $154603-21-1$ & $\mathrm{C}_{9} \mathrm{H}_{9} \mathrm{D}_{2} \mathrm{NO}_{2}$ & 168.09881 & 8.9 & TRC \\
\hline & $\begin{array}{l}\text { 1-(3-Pyridyl)-1,4-butane } \\
\text { diol }\end{array}$ & Diol & $\mathrm{HPB} \mathrm{D}_{2}$ & 76014-83-0 & $\mathrm{C}_{9} \mathrm{H}_{13} \mathrm{NO}_{2}$ & 168.10191 & 3.2 & TRC \\
\hline & $\begin{array}{l}\text { 1-(3-Pyridyl)-1-butanol- } \\
\text { 4-carboxylic acid }\end{array}$ & Hydroxy acid & $\mathrm{HPB} \mathrm{D}_{2}$ & $15569-97-8$ & $\mathrm{C}_{9} \mathrm{H}_{11} \mathrm{NO}_{3}$ & 182.08117 & 3.4 & TRC \\
\hline & $\begin{array}{l}\text { 1-(3-Pyridyl)-1-bu- } \\
\text { tanone-4-carboxylic } \\
\text { acid }\end{array}$ & OPBA & $\mathrm{HPB} \mathrm{D}_{2}$ & $4192-31-8$ & $\mathrm{C}_{9} \mathrm{H}_{9} \mathrm{NO}_{3}$ & 180.06552 & 9.0 & $\mathrm{TRC}$ \\
\hline & $\begin{array}{l}\text { 4-Hydroxy-1-(3- } \\
\text { pyridyl)-1-butanone }\end{array}$ & HPB & $\mathrm{HPB} \mathrm{D}_{2}$ & $59578-62-0$ & $\mathrm{C}_{9} \mathrm{H}_{11} \mathrm{NO}_{2}$ & 166.08626 & 8.9 & TRC \\
\hline \multirow[t]{2}{*}{ GSH } & $\begin{array}{l}\text { Glutathione reduced } \\
{ }^{13} \mathrm{C}_{2}^{15} \mathrm{~N}\end{array}$ & GSH ${ }^{13} \mathrm{C}_{2}{ }^{15} \mathrm{~N}$ & N/A & $815610-65-2$ & ${ }^{13} \mathrm{C}_{2}^{15} \mathrm{~N} \mathrm{C}_{8} \mathrm{H}_{17} \mathrm{~N}_{2} \mathrm{O}_{6} \mathrm{~S}$ & 311.09483 & 2.7 & Sigma-Aldrich \\
\hline & Glutathione reduced & $\mathrm{GSH}$ & $\mathrm{GSH}^{13} \mathrm{C}_{2}{ }^{15} \mathrm{~N}$ & $70-18-8$ & $\mathrm{C}_{10} \mathrm{H}_{17} \mathrm{~N}_{3} \mathrm{O}_{6} \mathrm{~S}$ & 308.09108 & 2.7 & Sigma-Aldrich \\
\hline
\end{tabular}

ISTDs isotopically labelled internal standards, SCBT Santa Cruz Biotechnology, TRC Toronto Research Chemicals

\subsection{Sample preparation}

The sample preparation workflow is shown in Fig. 2. Two extraction procedures were evaluated in parallel, based on either freeze-thawing or homogenization. Other than the extractions, all other steps were common to all samples.

After removal of the culture medium, cells were washed twice with $0.5 \mathrm{~mL}$ phosphate-buffered saline (PBS) without calcium chloride and magnesium chloride (Ref. D8537, Sigma).
For both extraction procedures, washed cell cultures were quenched with $150 \mu \mathrm{L}$ cold $\left(-20^{\circ} \mathrm{C}\right)$ methanol/ water $(4: 1 \mathrm{v} / \mathrm{v})$ containing the ISTDs and, for spiked samples, the standards. For the HepaRG ${ }^{\mathrm{TM}}$ spheroids, which were non-adherent, spheroids and solvent were transferred directly into an Eppendorf tube for further extraction. As the NHBE ALI tissues were adherent and not homogenous, tissues were harvested from the inserts after quenching by scraping into cold methanol/water, on ice. 


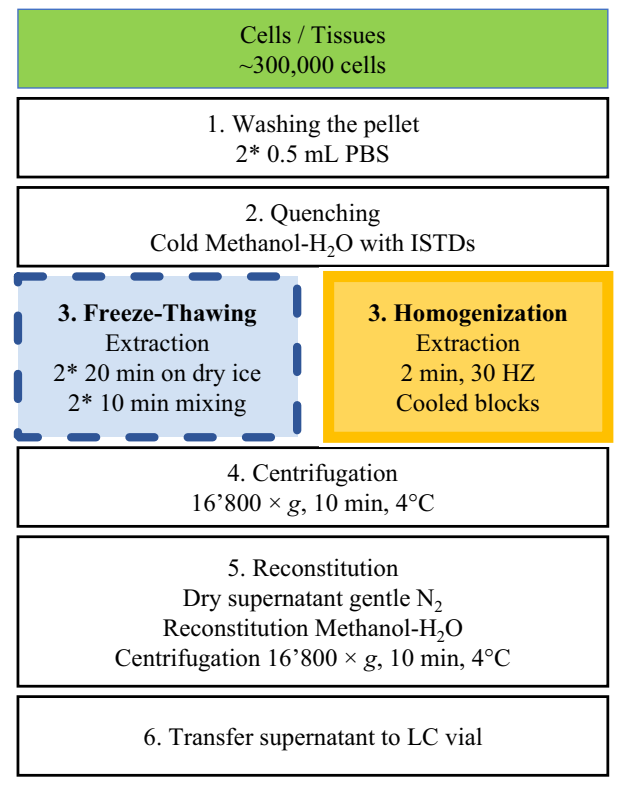

Fig. 2 Sample preparation workflow for freeze-thawing- and homogenization-based procedures. In both protocols, all steps were common, other than the extraction step (step 3). Because of the significant signal decrease or variability caused by drying-reconstitution, this step (step 5) was skipped in subsequent experiments

\subsubsection{Freeze-thawing extraction procedure}

After quenching, cells were frozen using dry ice for $20 \mathrm{~min}$ and then agitated for $10 \mathrm{~min}$ at $30{ }^{\circ} \mathrm{C}$ and $800 \mathrm{rpm}$ on a ThermoMixer C (Eppendorf, Schönenbuch, Switzerland). This process was repeated for two cycles.

\subsubsection{Homogenization extraction procedure}

Zirconium beads $(1.4 \mathrm{~mm})$ were added to the quenched cells, in Eppendorf tubes, and tubes were placed in cold homogenizer blocks (pre-cooled to $-20^{\circ} \mathrm{C}$ ). Samples were disrupted with a tissuelyser (QIAGEN, Hombrechtikon, Switzerland) for $2 \mathrm{~min}$ at $30 \mathrm{~Hz}$.

After extraction, to remove proteins and other cell debris, the extracts were centrifuged for $10 \mathrm{~min}$ at $16^{\prime} 800 \times g\left(4^{\circ} \mathrm{C}\right)$. Supernatants were transferred into vials $(60 \mu \mathrm{L})$ for LC-HRMS analysis.

For the drying-reconstitution step, instead of transferring the aliquot extracts to LC-HRMS vials directly, aliquots $(100 \mu \mathrm{L})$ were dried either under a gentle $\mathrm{N}_{2}$ stream at room temperature, or with a Speedvac at $30{ }^{\circ} \mathrm{C}$ (Christ, Osterode, Germany). The dried extracts were then reconstituted in $100 \mu \mathrm{L}$ methanol/water, (4:1 v/v). After centrifugation for $10 \mathrm{~min}$ at $4^{\circ} \mathrm{C}, 16^{\prime} 800 \times g$, supernatants were transferred into vials $(60 \mu \mathrm{L})$ for LC-HRMS analysis.

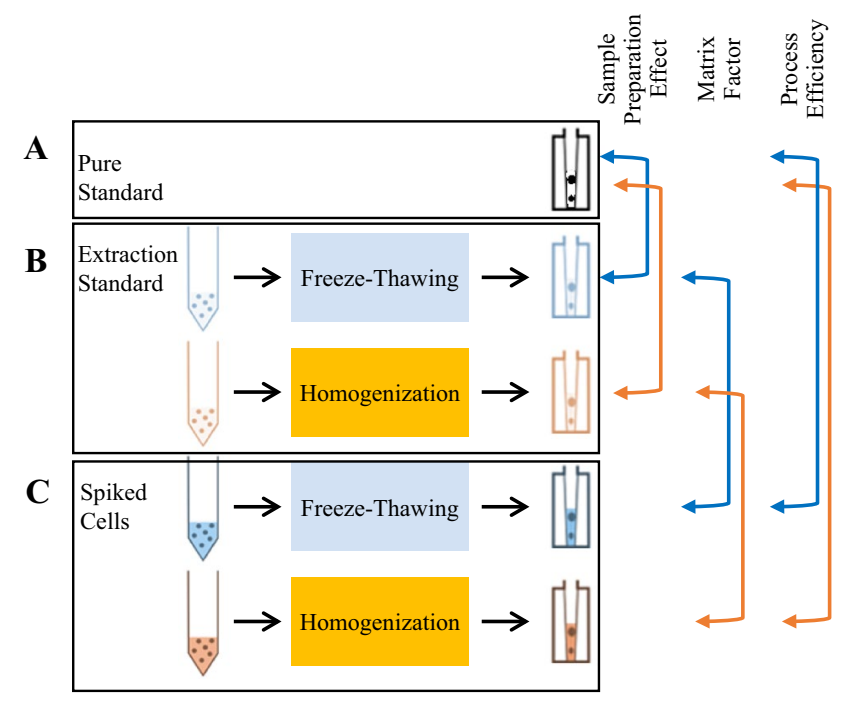

Fig. 3 Assay procedure to evaluate sample preparation methods by measuring sample preparation effect (b/a), matrix factor (c/b), and PE (c/a). Adapted from Mathon et al. (2013) and Noga et al. (2018)

\subsection{Experimental design}

To evaluate sample preparation, two extraction protocols (i.e. freeze-thawing or homogenization), were performed and compared for standard solutions and for each cell type (i.e. NHBE ALI tissues and HepaRG ${ }^{\mathrm{TM}}$ spheroids) (Figs. 2, $3)$. For both protocols the extraction solvent was a mixture of methanol-water $(80: 20 \mathrm{v} / \mathrm{v})$.

Concentrations measured in pure standard solutions (Fig. 3a) were compared to concentrations measured in standard solution going through the extractions (Fig. 3b), as well as concentrations measured in the spiked cells (Fig. 3c).

For each sub-group (e.g., pure standard, spiked HepaRG cells extracted with freeze-thaw procedure, ...), six replicates were prepared and injected once.

\subsection{LC-HRMS instrumentation}

All analyses were performed using an Accela 1250 ultrahigh performance LC system coupled to a Q-Exactive HRMS equipped with a heated electrospray ionization source (Thermo Fisher Scientific, Bremen, Germany).

Metabolite separation was performed using two complementary stationary phases in series, comprising a Biobasic anion-exchange column $(50 \mathrm{~mm} \times 2.1 \mathrm{~mm}, 5 \mu \mathrm{m}$ particle size, Thermo Scientific) followed by a Kinetex ${ }^{\circledR}$ pentafluorophenyl column $(150 \mathrm{~mm} \times 2.1 \mathrm{~mm}, 2.6 \mu \mathrm{m}$ particle size, $100 \AA$, Phenomenex, Basel, Switzerland). Samples were analyzed in positive ionization mode using $10 \mathrm{mM}$ ammonium formate buffer, $\mathrm{pH} 3.5$ (eluent $\mathrm{A}$ ), and methanol/water/ formic acid (95:5:0.1, v/v/v) (eluent B). The elution gradient was: held at $99 \% \mathrm{~A}$ for $2 \mathrm{~min}, 99 \% \mathrm{~A}$ to $55 \% \mathrm{~A}$ in $8 \mathrm{~min}$, 


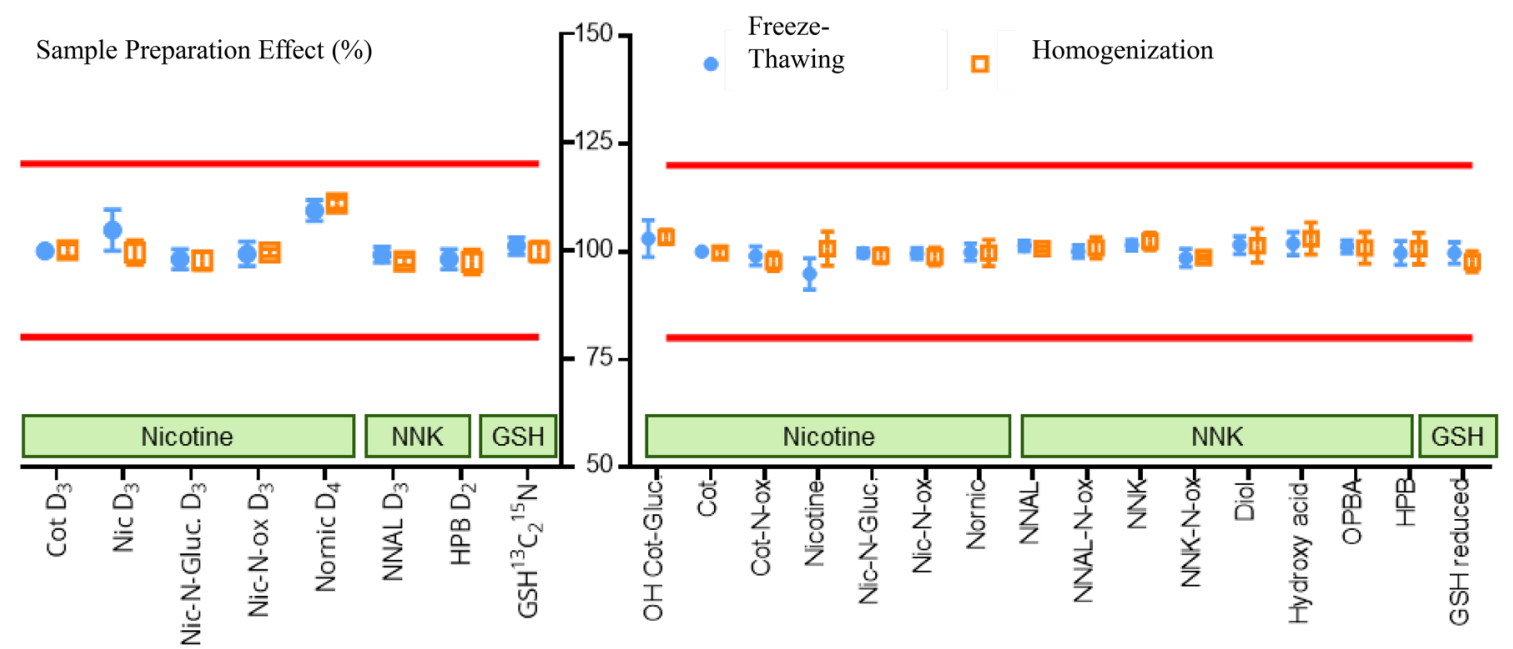

Fig. 4 Sample preparation effect of ISTDs and analytes with no matrices for three classes of metabolites: nicotine, NNK, and GSH. The data are mean sample preparation effect values for freeze-thawing-based extraction (filled dots) and homogenization-based extraction (unfilled squares). Bars show standard deviations $(n=6)$. Analyte recovery values were calculated based on analyte concentrations, as quantified using ISTDs. Cot

then decreasing to $0 \% \mathrm{~A}$ in 3 min and held at $100 \% \mathrm{~B}$ for $2 \mathrm{~min}$. Initial conditions were restored between samples by returning to $99 \%$ A over $1 \mathrm{~min}$, followed by equilibration for an additional $4 \mathrm{~min}$. The flow rate was $0.28 \mathrm{~mL} / \mathrm{min}$, the $\mathrm{LC}$ column oven was maintained at $40^{\circ} \mathrm{C}$, the injection volume was $1 \mu \mathrm{L}$ and the autosampler was maintained at $10^{\circ} \mathrm{C}$.

The HRMS system was calibrated and tuned according to the manufacturer's recommendations using the tune mix (REF 88323, ESI positive ion calibration solution, Fisher Scientific, Loughborough, UK) Nitrogen (purity > 99.999\%) was used as a sheath gas and auxiliary gas at a flow of 50 and 15 arbitrary units, respectively. Auxiliary gas and capillary temperatures were $350{ }^{\circ} \mathrm{C}$ and $300{ }^{\circ} \mathrm{C}$, respectively. Spray voltage was $3.00 \mathrm{kV}$.

Full scan acquisition mode $(\mathrm{m} / \mathrm{z} 60-900)$ at a mass resolving power of 70,000 (full width at half maximum, FWHM) at $\mathrm{m} / \mathrm{z} 200$ with an automatic gain control (AGC) target set at $5 \mathrm{e}^{5}$, and a maximum inject time of $150 \mathrm{~ms}$ was used. For tandem mass spectrometry (MS/MS) metabolite identification, the following conditions were used: data-dependent MS/MS top three of each scan at 17,500 (FWHM) at $\mathrm{m} / \mathrm{z} 200$, with an AGC target set at $5 \mathrm{e}^{4}$ and a maximum injection time of $150 \mathrm{~ms}$, with stepped normalized collision energies set at 25 , 50 , and $75 \mathrm{eV}$ with an isolation window of $1 \mathrm{~m} / \mathrm{z}$.

\subsection{Data processing and data analysis}

Metabolites detection, integration and quantification were done with TraceFinder 3.0 software (Thermo Fisher Scientific). cotinine, Diol 1-(3-pyridyl)-1,4-butane diol, Gluc. glucuronide, GSH glutathione, HPB 4-hydroxy-1-(3-pyridyl)-1-butanone-4,4, Hydroxy acid 1-(3-pyridyl)-1-butanol-4-carboxylic acid, Nic nicotine, NNAL 4-(methylnitrosamino)-1-(3-pyridyl)-1-butanol, NNK 4-(methylnitrosamino)-1-(3-pyridyl)-1-butanone, OPBA 1-(3-pyridyl)-1-butanone-4-carboxylic acid, $O x$ oxide

Calculations, tests (Holm-Sidak test, $\alpha 0.05$, heteroscedastic) and graphs (Figs. 4, 5) were performed using GraphPad Prism 7.0 software (GraphPad, CA, USA).

\section{Results and discussion}

For untargeted metabolomics analysis, an ideal preparation method would extract the maximum number of metabolites from a large number of samples with high precision. Sample preparation protocols can be influenced by cell culture properties (adherent or suspended, 2D or 3D cultures) as well as cell types, the physico-chemical properties of the metabolites, and the techniques used for their detection. 3D cell cultures were used for this investigation because they are now routinely used for toxicological assessment, and a new extraction approach was required compared with $2 \mathrm{D}$ cell cultures.

Understanding the metabolism pathways for xenobiotics, such as nicotine and its metabolites, the major markers for cigarette smoke exposure, requires precise metabolite analyses, either at individual time points or in kinetic studies. To evaluate the precision of a non-discriminative analytical approach for intracellular metabolite analysis, sample preparation effect, matrix factor, and PE were calculated for 16 metabolites of interest (i.e., nicotine, NNK, and their major metabolites) and eight ISTDs related to our research scope. Some ISTDs were used for more than one metabolite, based on their chemical and physical properties (Table 1). 


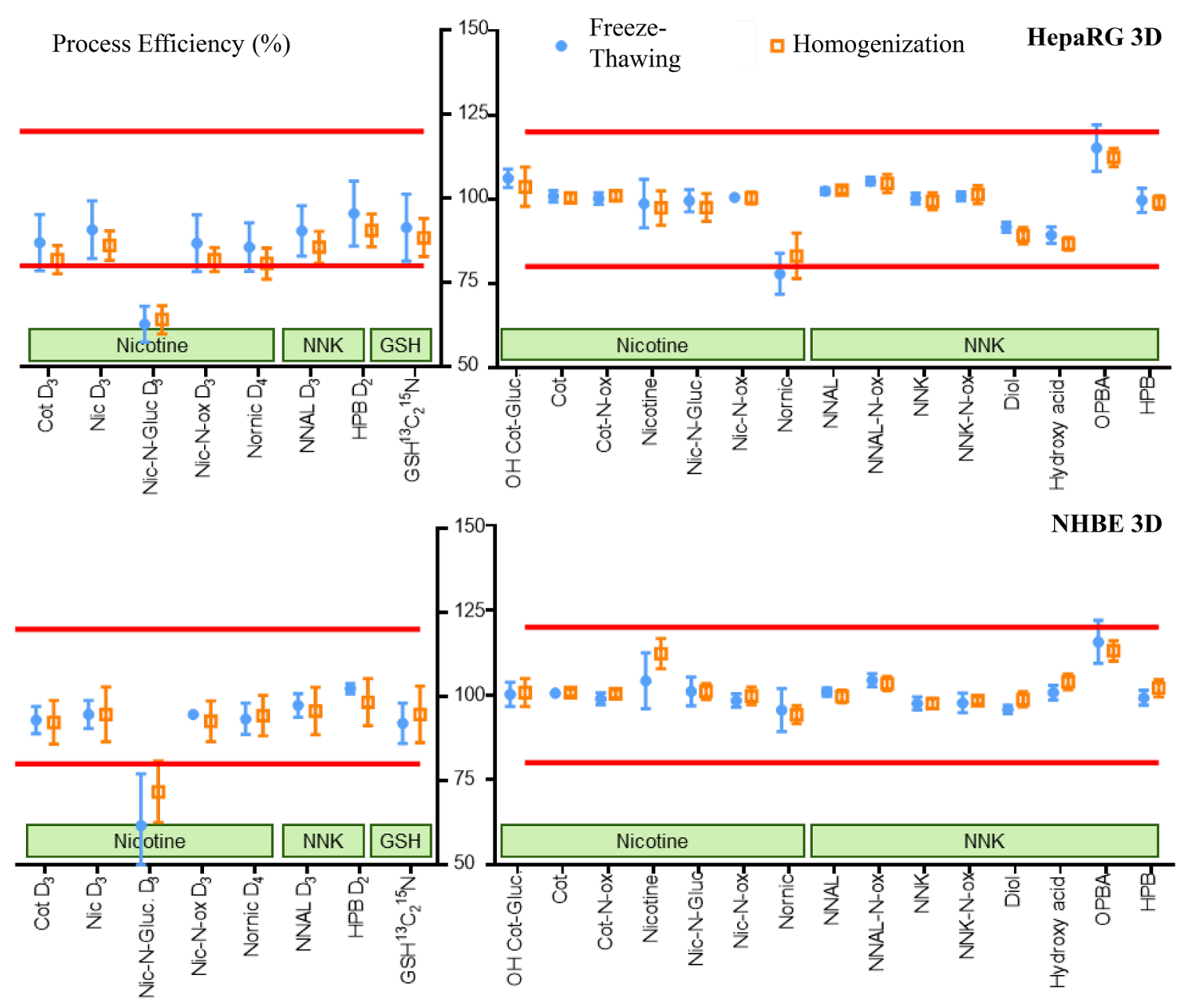

Fig. 5 PE (C/A) values for ISTDs and analytes for the three classes of metabolites: nicotine, NNK, and GSH. Samples were obtained from 3D tissues, HepaRG ${ }^{\mathrm{TM}}$ spheroids (upper) or NHBE ALI tissues (lower). Data are means for freeze-thawing-based extraction (filled dots) and homogenization-based extraction (unfilled squares). Bars show standard deviations $(n=6)$. Data for analytes were based on concentrations quantified using ISTDs. Cot

The addition of ISTDs, which were not present endogenously in the samples, enabled evaluation of any changes, including signal suppression or enhancement caused by sample preparation or analysis, in each extract (Broadhurst et al. 2018; Noga et al. 2018). As reported by Broadhurst et al. another advantage of including ISTDs in all samples was to enable real-time tracking of sample responses during data collection (mass accuracy and resolution, retention time, peak intensity, and peak shape). This is an advantage over the use of QC samples, which are not evaluated until the end of a batch or the entire study analysis (Broadhurst et al. 2018). Absolute quantification of compounds using ISTDs enables correction for procedural variations occurring at any time after the ISTDs were added to the sample. In our study, ISTDs were added at the beginning of sample preparation cotinine, Diol 1-(3-pyridyl)-1,4-butane diol, Gluc. glucuronide, GSH glutathione, HPB 4-hydroxy-1-(3-pyridyl)-1-butanone-4,4, Hydroxy acid 1-(3-pyridyl)-1-butanol-4-carboxylic acid, Nic nicotine, NNAL 4-(methylnitrosamino)-1-(3-pyridyl)-1-butanol, NNK 4-(methylnitrosamino)-1-(3-pyridyl)-1-butanone, OPBA 1-(3-pyridyl)-1-butanone-4-carboxylic acid, $O x$ oxide

and during cell quenching and extraction to address any post-harvesting changes in target metabolites. Results are presented for analyte concentrations quantified using the ISTDs as well as for the ISTD responses themselves.

A rapid, simple sample preparation method, performed at a low temperature to minimize changes in metabolites, was evaluated using two different extraction methods.

\subsection{Assay procedure}

To evaluate sample preparation and the analytical method, selected metabolites were injected as pure standards, as standards without matrix undergoing sample extraction procedures, and as standards with matrix undergoing sample extraction procedures (Fig. 3). One advantage of cell culture 
is that the environment is controlled, limiting sample-tosample variations and enabling robust statistics with a limited number of replicates ( $n=6$ in this study) (Dudzik et al. 2018).

To evaluate sample preparation effects, results obtained with pure standard solutions, containing the 16 metabolites and the eight ISTDs, were compared with those for the same solution subjected to two sample preparation procedures, freeze-thawing and homogenization extraction procedures, without matrix (Fig. 3).

In an analogous manner, the impact of the matrix, calculated as a matrix factor, was determined by comparing the results obtained for extracted standard solutions with those of metabolites and ISTDs spiked on cells during the quenching step (Fig. 3).

Finally, PE was determined by comparing results from injected pure standard solutions with those from spiked cell samples undergoing the complete sample preparation process (Fig. 3).

For endogenous metabolites such as GSH, always present in the intracellular metabolome, PE was measured using the ISTD only.

\subsection{Cell washing, quenching, and extraction}

To evaluate the intracellular metabolome, medium was removed, and cells were washed to remove medium components known to affect the cellular metabolome (Daskalaki et al. 2018). The washed 3D cell cultures were then quenched. During all processing, cells were cooled on either ice or dry ice.

For the adherent and not homogenous NHBE ALI tissues, cells were harvested from the inserts by scraping into cold methanol/water, on ice. Scraping limited metabolite leakage, as previously demonstrated with EDTA treatment (Dudzik et al. 2018). Samples were then directly extracted using two freeze-thaw cycles or one homogenization cycle, as described in Sect. 2.5.

\subsection{Drying and reconstitution}

Drying and reconstitution is commonly used to concentrate samples in order to increase the number of metabolites detected. Additionally, samples can be reconstituted in solvents other than the extraction solution for compatibility with the analytical method.

The impact of the drying step used to concentrate the extracts on metabolite detection was quantified by reconstituting the samples in the same solvent (e.g., the extraction mixture [methanol/water, 4:1 v/v]) (Fig. 2). The concentration procedure substantially decreased the responses for nicotine, nornicotine, GSH, and their corresponding ISTDs. For instance, after overnight drying nornicotine $\mathrm{D}_{4}$ showed a very good relative standard deviation (RSD) in the calibration solutions ( $\mathrm{RSD}=3 \%$ ), while for samples been through the extraction processes (freeze-thawing or homogenization) the RSD were high (between 24 and 72\%), for both cell types. Additionally, the nicotine $\mathrm{D}_{3}$ PEs were low (between $18 \%$ for HepaRG ${ }^{\mathrm{TM}}$ spheroids after homogenization, to $54 \%$ for NHBE ALI tissues after freeze-thawing). While drying overnight led to consistent signal decreases in all samples, a duration of $2 \mathrm{~h}$ resulted in high variability in the data. For example, nicotine $\mathrm{D}_{3}$ was not detected in samples after overnight drying, while nicotine $\mathrm{D}_{3}$ was detected in $61 \%$ of the samples dried $2 \mathrm{~h}$.

High response variability or absence of the ISTD drastically impacts quantification of the metabolites, if they are detected. Because of the significant signal decrease or variability caused by drying, this step was not included in subsequent experiments. After extraction and centrifugation, samples were instead directly transferred to LC-HRMS vials for analyses (Fig. 2).

If a drying step is required to concentrate an extract or modify solvent composition, ISTDs must be added to correct for any metabolite changes caused by drying.

\subsection{Method performance}

To evaluate sample preparation effect without matrix, the ratio between compound responses observed in a standard solution processed by the two extraction procedures were compared with those in pure standard solutions (Fig. 3).

There were no significant differences between the two extraction procedures in analysis of the ISTDs (Holm-Sidak test, $\alpha 0.05$, heteroscedastic). The mean sample preparation effect values $(\mathrm{n}=6)$ for the ISTDs were between $97.3 \pm 2.8 \%$ (for 4-hydroxy-1-(3-pyridyl)-1-butanone $\mathrm{D}_{2}$ [HPB-D $\left.\mathrm{D}_{2}\right]$ ) and $110.8 \pm 1.2 \%$ (for nornicotine $\mathrm{D}_{4}$ ), both after the homogenization process, as shown in Fig. 4 (left). The analytes were quantified using their corresponding ISTDs (Table 1), and no significant differences were observed between the two extraction processes (Holm-Sidak test, $\alpha 0.05$, heteroscedastic). The mean sample preparation effect values $(n=6)$ for the analytes were between $94.8 \pm 3.6 \%$ for nornicotine (after the freeze-thawing process) and $103.3 \pm 1.6 \%$ for 3-hydroxycotinine- $O$-glucuronide (OH Cot-Gluc, after the homogenization process), as shown in Fig. 4 (right).

As detailed above, without matrix, sample preparation recoveries were good for ISTDs and analytes, with accurate, reproducible results and no significant differences between the two extraction procedures.

To evaluate the impact of extraction procedures on metabolites from NHBE ALI tissues and HepaRG ${ }^{\mathrm{TM}}$ spheroids, PE was calculated by comparing results from spiked cell cultures to those from pure standard solutions (Fig. 3). 
For the ISTDs, PE values were calculated by dividing the area obtained for each ISTD from spiked matrices by that from pure standard solutions. For HepaRG ${ }^{\mathrm{TM}}$ spheroids, $\mathrm{PE}$ values were between $62.7 \pm 5.3 \%$ (nicotine- $N$-glucuronide $\mathrm{D}_{3}$, Nic- $N$-Gluc. $\left.\mathrm{D}_{3}\right)$ and $95.5 \pm 9.9 \%\left(\mathrm{HPB} \mathrm{D}_{2}\right)$ using homogenization (Fig. 5, upper left). For NHBE ALI tissues, Nic- $N$-Gluc. $\mathrm{D}_{3}$ was detected with lowest efficiencies $(61.7 \pm 15.3 \%$ and $71.7 \pm 9.1 \%$ for homogenization and freeze-thawing, respectively), as shown in Fig. 5 (lower left).

For the analytes, PE values were calculated using concentrations quantified with ISTDs in spiked matrices divided by those measured in pure standard solutions. Of the metabolites, nornicotine had the lowest PE value in HepaRG ${ }^{\mathrm{TM}}$ spheroids $(83.2 \pm 6.7 \%$ and $77.8 \pm 6.0 \%$ for homogenization and freeze-thawing, respectively) as well as in NHBE ALI tissues $(94.2 \pm 2.6 \%$ and $95.5 \pm 6.3 \%$ for homogenization and freeze-thawing, respectively) (Fig. 5, right). The highest PE values were obtained in both matrices and extraction procedures for 1-(3-pyridyl)-1-butanone-4-carboxylic acid (OPBA) in NHBE ALI tissues $(113.0 \pm 3.0 \%$ and $115.7 \pm 6.3 \%$ for homogenization and freeze-thawing, respectively) and in HepaRG ${ }^{\mathrm{TM}}$ spheroids $(112.3 \pm 2.7 \%$ and $115.2 \pm 6.9 \%$ for homogenization and freeze-thawing, respectively) (Fig. 5, right).

The results for OPBA analysis were explained by the use of a non-isotopologue ISTD, HPB $\mathrm{D}_{2}$, a stable isotopelabelled compound with a molecular structure that is close, but not exactly the same. When the ISTDs and analytes were isotopologues, the analyte PE values were nearly $100 \%$, as shown for nicotine- $N$-glucuronide in HepaRG ${ }^{\mathrm{TM}}$ spheroids $(97.5 \pm 4.1 \%$ and $99.5 \pm 3.3 \%$ for homogenization and freeze-thawing, respectively) and in NHBE ALI tissues $(101.0 \pm 2.4 \%$ and $101.0 \pm 4.2 \%$ for homogenization and freeze-thawing, respectively). In contrast, Nic- $\mathrm{N}$ Gluc $\mathrm{D}_{3}$ analysis had the lowest $\mathrm{PE}$ values in homogenized HepaRG $^{\mathrm{TM}}$ spheroids $(62.7 \pm 5.3 \%)$ and in NHBE ALI tissues $(61.7 \pm 15.3 \%$ and $71.7 \pm 9.1 \%$ for homogenization and freeze-thawing, respectively). Such results illustrated the importance of using appropriate ISTDs to correct for signal losses or enhancement during sample preparation and data acquisition. Without ISTDs, the true metabolite concentration cannot be determined (Broadhurst et al. 2018).

As shown in Fig. 3, the PE value is a combination of sample preparation effect and matrix factor. Our results indicated that our sample preparation procedures yielded accurate and reproducible results for all metabolites, independently of the extraction process used. The matrix effects (not shown) showed similar trends as the PE values. Thus the loss or enhancement of some ISTDs and metabolites detected, based on PE values, were caused by the presence of the matrices.

For extraction of cell cultures, monolayers, or tissues, use of freeze-thawing cycle(s) or physical disruption (homogenization or ultrasonication) have been reported in literature without studies comparing these methods. Here, we compared freeze-thawing and homogenization extraction procedures to evaluate the most suitable sample preparation methods, based on recovery, efficiency, and repeatability. The two extraction procedures showed no significant differences in recovery, repeatability, and efficiency (Figs. 4, 5).

For sample preparation, extraction by homogenization required only $2 \mathrm{~min}$, compared with approximatively $1 \mathrm{~h}$ required for extraction by freeze-thawing. However, the presence of beads used for homogenization required an additional transfer step to obtain clean extracts, separated from beads, protein, and cell debris. Without a drying-reconstitution step, both sample preparation techniques are simple and fast and comparable with "dilute and shoot" methods applied for the analysis of homogenous matrices (e.g., urine). Rapid, simple measures should decrease the risk of changes in the metabolome during sample preparation.

\section{Conclusions}

These results demonstrated the importance of using ISTDs to correct for signal losses or enhancements caused by the presence of matrices. We showed that structurally related ISTDs can enable partial compensation for metabolite losses or enhancements, while isotopologue ISTDs enable full compensation for such artifacts. Additionally, the resulting ability to absolutely quantify analytes will improve interstudy and inter-laboratory comparisons.

Among the tobacco-related metabolites evaluated, the results for some were strongly affected by a drying-reconstitution step, some metabolites or ISTDs such as nicotine and nicotine $\mathrm{D}_{3}$ were not always detected after a drying step. For other compounds such as nornicotine, the drying step induced high RSD and low recoveries. By eliminating this step, favorable recoveries were obtained for those analytes. Furthermore, for all compounds assessed, there were no significant differences between homogenization- and freezethawing-based extractions.

The sample preparation methods evaluated here were rapid, easily performed, and gave repeatable results due to the ISTDs' for complex adherent and suspended 3D cell cultures. The metabolite list is flexible, expendable and customizable according to study needs; thus the protocols and method presented herein can be useful for metabolomics investigation.

Author contribution $\mathrm{CM}$ and $\mathrm{AK}$ conceived and designed the research. DB conducted cell culture experiments. CM performed the experiments, data processing and data analysis. $\mathrm{CM}$ wrote the manuscript. All authors read and approved the manuscript. 
Funding Philip Morris International is the sole source of funding and sponsor of this research.

\section{Compliance with ethical standards}

Conflict of interest All authors are employees of Philip Morris International. All authors declare that they have no conflict of interest.

Ethical approval This article does not contain any studies with human and/or animal participants performed by any of the authors.

Open Access This article is distributed under the terms of the Creative Commons Attribution 4.0 International License (http://creativeco mmons.org/licenses/by/4.0/), which permits unrestricted use, distribution, and reproduction in any medium, provided you give appropriate credit to the original author(s) and the source, provide a link to the Creative Commons license, and indicate if changes were made.

\section{References}

Alepee, N., Bahinski, A., Daneshian, M., De Wever, B., Fritsche, E., Goldberg, A., et al. (2014). State-of-the-art of 3D cultures (organs-on-a-chip) in safety testing and pathophysiology. Altex, 31, 441-477.

Baxter, A., Thain, S., Banerjee, A., Haswell, L., Parmar, A., Phillips, G., et al. (2015). Targeted omics analyses, and metabolic enzyme activity assays demonstrate maintenance of key mucociliary characteristics in long term cultures of reconstituted human airway epithelia. Toxicology in Vitro, 29, 864-875.

Bi, H., Krausz, K. W., Manna, S. K., Li, F., Johnson, C. H., \& Gonzalez, F. J. (2013). Optimization of harvesting, extraction, and analytical protocols for UPLC-ESI-MS-based metabolomic analysis of adherent mammalian cancer cells. Analytical and Bioanalytical Chemistry, 405, 5279-5289.

Bovard, D., Iskandar, A., Luettich, K., Hoeng, J., \& Peitsch, M. C. (2017). Organs-on-a-chip. Toxicology Research and Application, $1,1-16$.

Broadhurst, D., Goodacre, R., Reinke, S. N., Kuligowski, J., Wilson, I. D., Lewis, M. R., et al. (2018). Guidelines and considerations for the use of system suitability and quality control samples in mass spectrometry assays applied in untargeted clinical metabolomic studies. Metabolomics, 14, 72.

Cala, M. P., \& Meesters, R. J. (2017). Comparative study on microsampling techniques in metabolic fingerprinting studies applying gas chromatography-MS analysis. Bioanalysis, 9, 1329-1340.

Cuykx, M., Mortele, O., Rodrigues, R. M., Vanhaecke, T., \& Covaci, A. (2017). Optimisation of in vitro sample preparation for LC-MS metabolomics applications on HepaRG cell cultures. Analytical Methods, 9, 3704-3712.

Daskalaki, E., Pillon, N. J., Krook, A., Wheelock, C. E., \& Checa, A. (2018). The influence of culture media upon observed cell secretome metabolite profiles: The balance between cell viability and data interpretability. Analytica Chimica Acta, 1037, 338-350.

Dettmer, K., Nürnberger, N., Kaspar, H., Gruber, M. A., Almstetter, M. F., \& Oefner, P. J. (2011). Metabolite extraction from adherently growing mammalian cells for metabolomics studies: Optimization of harvesting and extraction protocols. Analytical and Bioanalytical Chemistry, 399, 1127-1139.

Drouin, N., Rudaz, S., \& Schappler, J. (2018). Sample preparation for polar metabolites in bioanalysis. Analyst, 143, 16-20.
Dudzik, D., Barbas-Bernardos, C., Garcia, A., \& Barbas, C. (2018). Quality assurance procedures for mass spectrometry untargeted metabolomics: A review. Journal of Pharmaceutical and Biomedical Analysis, 147, 149-173.

Fathi, F., Brun, A., Rott, K. H., Cobra, P. F., Tonelli, M., Eghbalnia, H. R., et al. (2017). NMR-based identification of metabolites in polar and non-polar extracts of avian liver. Metabolites, 7, 61/1-61/9.

Griffith, C. M., Morgan, M. A., Dinges, M., Mathon, C., \& Larive, C. K. (2018). Metabolic profiling of chloroacetanilide herbicides in earthworm coelomic fluid using (1)H NMR and GC-MS. Journal of Proteome Research, 17, 2611-2622.

Groell, F., Jordan, O., \& Borchard, G. (2018). In vitro models for immunogenicity prediction of therapeutic proteins. European Journal of Pharmaceutics and Biopharmaceutics, 130, $128-142$.

Hartung, T., FitzGerald, R. E., Jennings, P., Mirams, G. R., Peitsch, M. C., Rostami-Hodjegan, A., et al. (2017). Systems toxicology: Real world applications and opportunities. Chemical Research in Toxicology, 30, 870-882.

Iskandar, A. R., Mathis, C., Schlage, W. K., Frentzel, S., Leroy, P., Xiang, Y., et al. (2017). A systems toxicology approach for comparative assessment: Biological impact of an aerosol from a candidate modified-risk tobacco product and cigarette smoke on human organotypic bronchial epithelial cultures. Toxicology in Vitro, 39, 29-51.

Ivanisevic, J., Zhu, Z.-J., Plate, L., Tautenhahn, R., Chen, S., O’Brien, P. J., et al. (2013). Toward 'omic scale metabolite profiling: A dual separation-mass spectrometry approach for coverage of lipid and central carbon metabolism. Analytical Chemistry, 85, 6876-6884.

Karp, P. H., Moninger, T. O., Weber, S. P., Nesselhauf, T. S., Launspach, J. L., Zabner, J., et al. (2002). An in vitro model of differentiated human airway epithelia. Methods for establishing primary cultures. Methods in Molecular Biology, 188, 115-137.

Kogel, U., Titz, B., Schlage, W. K., Nury, C., Martin, F., Oviedo, A., et al. (2016). Evaluation of the Tobacco Heating System 2.2. Part 7: Systems toxicological assessment of a mentholated version revealed reduced cellular and molecular exposure effects compared with mentholated and non-mentholated cigarette smoke. Regulatory Toxicology and Pharmacology, 81, S123-S138.

Konstantinou, E., Fotopoulou, F., Drosos, A., Dimakopoulou, N., Zagoriti, Z., Niarchos, A., et al. (2018). Tobacco-specific nitrosamines: A literature review. Food and Chemical Toxicology, 118, 198-203.

Martinovic, T., Srajer Gajdosik, M., \& Josic, D. (2018). Sample preparation in foodomic analyses. Electrophoresis, 39, 1527-1542.

Mathon, C., Bongard, B., Duret, M., Ortelli, D., Christen, P., \& Bieri, S. (2013). Analysis of the neurotoxin anisatin in star anise by LC-MS/MS. Food Additives \& Contaminants, Part A: Chemistry, Analysis, Control, Exposure \& Risk Assessment, 30, 1598-1605.

Noga, M. J., Zielman, R., van Dongen, R. M., Bos, S., Harms, A., Terwindt, G. M., et al. (2018). Strategies to assess and optimize stability of endogenous amines during cerebrospinal fluid sampling. Metabolomics, 14, 44.

Pezzulo, A. A., Starner, T. D., Scheetz, T. E., Traver, G. L., Tilley, A. E., Harvey, B. G., et al. (2011). The air-liquid interface and use of primary cell cultures are important to recapitulate the transcriptional profile of in vivo airway epithelia. American Journal of Physiology. Lung Cellular and Molecular Physiology, 300, L25-L31.

Pinu, F. R., Villas-Boas, S. G., \& Aggio, R. (2017). Analysis of intracellular metabolites from microorganisms: Quenching and extraction protocols. Metabolites, 7, 53/1-53/20.

Ravi, M., Paramesh, V., Kaviya, S. R., Anuradha, E., \& Solomon, F. D. (2015). 3D cell culture systems: Advantages and applications. Journal of Cellular Physiology, 230, 16-26.

Römisch-Margl, W., Prehn, C., Bogumil, R., Röhring, C., Suhre, K., \& Adamski, J. (2012). Procedure for tissue sample preparation and 
metabolite extraction for high-throughput targeted metabolomics. Metabolomics, 8, 133-142.

Sapcariu, S. C., Kanashova, T., Weindl, D., Ghelfi, J., Dittmar, G., \& Hiller, K. (2014). Simultaneous extraction of proteins and metabolites from cells in culture. MethodsX, 1, 74-80.

Takahashi, Y., Hori, Y., Yamamoto, T., Urashima, T., Ohara, Y., \& Tanaka, H. (2015). 3D spheroid cultures improve the metabolic gene expression profiles of HepaRG cells. Bioscience Reports, 35, e00208.

Töpfer, N., Kleessen, S., \& Nikoloski, Z. (2015). Integration of metabolomics data into metabolic networks. Frontiers in Plant Science, $6,49$.

Ulmer, C. Z., Yost, R. A., Chen, J., Mathews, C. E., \& Garrett, T. J. (2015). Liquid chromatography-mass spectrometry metabolic and lipidomic sample preparation workflow for suspension-cultured mammalian cells using Jurkat T lymphocyte cells. Journal of Proteomics \& Bioinformatics, 8, 126-132.

Vinaixa, M., Schymanski, E. L., Neumann, S., Navarro, M., Salek, R. M., \& Yanes, O. (2016). Mass spectral databases for LC/MS- and
GC/MS-based metabolomics: State of the field and future prospects. TrAC, Trends in Analytical Chemistry, 78, 23-35.

Worley, B., \& Powers, R. (2013). Multivariate analysis in metabolomics. Current Metabolomics, 1, 92-107.

Zanetti, F., Titz, B., Sewer, A., Lo Sasso, G., Scotti, E., Schlage, W. K., et al. (2017). Comparative systems toxicology analysis of cigarette smoke and aerosol from a candidate modified risk tobacco product in organotypic human gingival epithelial cultures: A 3-day repeated exposure study. Food and Chemical Toxicology, 101, $15-35$.

Publisher's Note Springer Nature remains neutral with regard to jurisdictional claims in published maps and institutional affiliations. 